\title{
Bedtime Single-Dose Prednisolone in Clinically Stable Rheumatoid Arthritis Patients
}

\author{
Owlia Mohammad Bagher, ${ }^{1}$ Mehrpoor Golbarg, ${ }^{1}$ and Modares Mosadegh Moneyreh ${ }^{2}$ \\ ${ }^{1}$ Department of Internal Medicine, Shahid Sadoughi University of Medical Sciences, Yazd, Iran \\ ${ }^{2}$ Department of Pharmacology, Shahid Sadoughi University of Medical Sciences, Yazd, Iran
}

Correspondence should be addressed to Owlia Mohammad Bagher, bagherowlia@gmail.com

Received 26 August 2011; Accepted 22 December 2011

Academic Editors: T. Hirano, J. C. Laguna, and T. W. Stone

Copyright (C) 2012 Owlia Mohammad Bagher et al. This is an open access article distributed under the Creative Commons Attribution License, which permits unrestricted use, distribution, and reproduction in any medium, provided the original work is properly cited.

\begin{abstract}
Introduction. Sign and symptoms of rheumatoid arthritis have circadian rhythms and are more prominent in the morning. Timing of glucocorticoid administration may be important with respect to the natural secretion of endogenous glucocorticoids. Herein, we intended to test the hypothesis that bedtime administration of prednisolone could be more efficient in controlling signs and symptoms in patients with RA. Material and Methods. Sixty patients with stable disease were treated with single dose prednisolone at 8 a.m. for the first three months and thereafter with similar dose at 10 PM for the next three months (before-after method). We compared fatigue scores, morning stiffness and pain scores, Clinical Disease Activity Indices, erythrocyte sedimentation rates, C Reactive Protein, and profile of adverse effects. Results. The mean of morning stiffness, fatigue scores, CRP and CDAI decreased statistically when prednisolone was administrated at 10 p.m. The means of pain scores and ESR were also decreased when the patients took prednisolone at night, without significant statistical difference. Conclusion. Administration of low-dose oral prednisolone could reduce disease activity scores in morning in clinically stable patients with RA. So it could be supposed that administrating bedtime prednisolone may permit the smallest possible dose.
\end{abstract}

\section{Introduction}

In rheumatoid arthritis (RA), a circadian rhythm of disease activity has been well documented [1]. Morning stiffness, joint pain, and swelling (one of the RA classification criteria) are worse in morning, which could be explained by diurnal variations in the metabolism or secretion of endogenous cortisol and cytokines, especially IL6 [2]. Corticotropinreleasing hormone $(\mathrm{CRH})$ release leads to the pituitary production of corticotrophin (ACTH), followed by glucocorticoid secretion by the adrenal cortex. These components constitute the hypothalamic-pituitary-adrenocortical (HPA) axis, which has a circadian rhythm [3]. The ACTH level varies during the day due to its pulsatile secretion. As a result, in physiologic conditions, plasma ACTH and serum cortisol concentrations are highest at about the time of waking in the morning (at $8 \mathrm{AM}$ ), decrease irregularly during the day, are low in the evening, and reach their nadir after beginning sleep (at $2 \mathrm{AM}$ ) [4]. In RA patients, impaired cortisol secretion to ACTH has been described, supporting the concept of a relative adrenal glucocorticoid insufficiency [5]. Moreover, IL6 is also considered to be responsible for stimulating the production of acute phase of proteins, and increased levels of it have been reported in serum and synovial fluid in RA. Increased levels of IL6 were found from $2 \mathrm{AM}$ to $7 \mathrm{AM}$ in RA patients, which has been associated with a flare in disease activity in early morning $[6,7]$. Therefore, diurnal variations in the clinical signs and symptoms of RA may be partly due to changes in the cortisol and proinflammatory cytokine levels.

Glucocorticoids (GCs) are among the most frequently used drugs in treating RA, and most commonly are used to suppress inflammation and pain. This effect is brought about by their anti-inflammatory and immunosuppressive properties [8]. But HPA axis support is a crucial rule of lowdose GCs in stable RA.

When a potentially harmful drug such as a GC is prescribed, a balance between side effects and therapeutic 
TABLE 1: Characteristics of the patients at initiation of study.

\begin{tabular}{lc}
\hline Age $($ year $)($ mean \pm SD) & $20-67(46 \pm 11.09)$ \\
\hline Female/male & $51 / 9$ \\
Duration of disease $($ year $)($ mean \pm SD) & $1-40(7.1 \pm 7.5)$ \\
Number of involved joints (mean \pm SD) & $0-8(4.1 \pm 1.78)$ \\
Mean of fatigue score & $2-8(4.6 \pm 1.90)$ \\
Mean of pain score & $0-2(1.18 \pm 0.46)$ \\
Mean of morning stiffness score & $1-3(1.91 \pm 0.67)$ \\
Mean of ESR & $3-60(24.11 \pm 14.01)$ \\
Mean of CRP & $6-24(11.37 \pm 8.31)$ \\
Mean of CDAI & $4-9(6.56 \pm 1.35)$ \\
Mean of prednisolone dose & $5-7.5(5.125 \pm 0.549)$ \\
\hline
\end{tabular}

CDAI: Clinical Disease Activity Indices, ESR: erythrocyte sedimentation rate, CRP: C-reactive protein.

TABle 2: Profile of DMARDs used in the patients.

\begin{tabular}{lc}
\hline DMARDs & Number (percent) \\
\hline HCQ or SSZ or MTX & $13(21.7)$ \\
HCQ + MTX & $32(53.3)$ \\
MTX + SSZ & $4(6.7)$ \\
HCQ + SSZ & $1(1.7)$ \\
MTX + HCQ + CsA & $1(1.7)$ \\
MTX + SSZ + CsA & $1(1.7)$ \\
MTX + SSZ + HCQ & $8(13.3)$ \\
\hline
\end{tabular}

MTX: methotrexate, HCQ: hydroxychloroquine, SSZ: sulfasalazine, CsA: cyclosporine A.

benefits is attempted, and it is prudent to administer the minimal dose for the shortest duration to avoid adverse effects [9]. With assumption of minimizing interference with the normal diurnal hormonal pattern, in usual practice, in single-dose schedule, prednisolone is administered in the morning. It seems that administration of prednisolone at bedtime, which precedes the circadian flare of inflammatory activity, can potentially better control disease activity in somewhat lower doses [10].

In order to reduce the chance of HPA suppression, we should use the lowest dose of short half-life GCs, preferably in alternate fashion. Accordingly, using the drug at the optimal time may permit the smallest possible dose to be used. Prescribing GCs at night may in part lead to the suppression of an explosive surge of cytokines between 2 AM and 7 AM by better HPA axis support, so it is assumed that when a low dose GC is used for relief of morning stiffness, night administration could be more effective than an equivalent dose given in the morning [5].

In this study, we decided to test the hypothesis that administration of low-dose prednisolone at bedtime could be more effective in control of disease activity and morning symptoms.

\section{Material and Methods}

Sixty patients who fulfilled the criteria of the 2010 American College of Rheumatology [11] for RA were included in this study from April to September 2008. All patients were referred to the Yazd Rheumatology Center and were studied after obtaining informed consents and approval of the local ethics committee according to the Declaration of Helsinki. At the start of the study, all of the patients had been on long treatment with a single daily dose of prednisolone $(\leq 7.5 \mathrm{mg} /$ day $)$ and one or more disease modifying antirheumatic drugs (DMARDs).

Those who were taking drugs other than nonsteroidal anti-inflammatory drugs (NSAIDs) and DMARDs and those with any comorbid conditions such as diabetes, hypertension, and other chronic diseases were excluded.

$5 \mathrm{mg}$ prednisolone was reinstituted for the patients in the morning (8 AM) for the first three months, and, for the next three months, the patients received $5 \mathrm{mg}$ prednisolone at 10 PM (before-after method). No change in the DMARDs was scheduled during the study. Patients took their drug at 10 PM to prevent interference with their sleep.

A questionnaire including age, gender, the number of involved and tender joints, pain score (none $=0$, mild $=1$, moderate $=2$, severe $=3$ ), fatigue score (none $=0$, mild $=1$ 3 , moderate $=4-6$, severe $=7-10)$, morning stiffness score $(0$ = none, $1 \leq 30 \mathrm{~min}, 2=30-60 \mathrm{~min}, 3=1-2 \mathrm{~h}, 4=2-4 \mathrm{~h}, 5 \geq$ $4 \mathrm{~h}$ and $6=$ all day), erythrocyte sedimentation rates (ESR), C reactive protein (CRP), and supposed adverse effects profile of tachycardia, nocturia, sleep disturbance, infection, and blood pressure disturbance was designed by the authors on the basis of similar studies. ESR and CRP were measured at the beginning of the study and end of the first and second trimester. The clinical data were collected at the end of each month during the study.

We used the Clinical Disease Activity Index (CDAI) [12], a numerical index that is calculated by summing the number of tender and swollen joints using the 28-joint count and the patient and physician global assessments on a $10 \mathrm{~cm}$ visual analogue scale. The CDAI ranges from 0 to 76 . The cut points for remission and various degrees of disease activity are as follows:

(i) remission $\leq 2.8$,

(ii) low disease activity $\leq 10$,

(iii) moderate disease activity $\leq 22$,

(iv) high disease activity $>22$.

Means of each variable within the first and second trimester and at the end of each trimester were compared.

\section{Statistical Analysis}

Data are presented as mean \pm standard deviation of the mean. Statistical significance was calculated by nonparametric Wilcoxon's signed ranks test using SPSS version 11.5 (SPSS Inc., Chicago, IL, USA) for Windows. A $P$ value $<0.05$ was considered as significant difference.

\section{Results}

Sixty patients, nine male (15\%) and fifty-one female (85\%), with a mean age of $46 \pm 11.09$, were included in the 
TABLE 3: Comparison of mean of variables between two groups.

\begin{tabular}{lccc}
\hline Variable & Morning group \pm SD $($ min-max $)$ & Bedtime group \pm SD $(\min -\mathrm{max})$ & $P$ value \\
\hline Fatigue score $^{\mathrm{b}}$ & $4.58 \pm 1.87(1-8)$ & $3.58 \pm 2.17(0-7)$ & 0.000 \\
Pain score $^{\mathrm{c}}$ & $1.000 \pm 0.52(0-2)$ & $0.88 \pm 0.52(0-2)$ & 0.146 \\
Morning stiffness score $^{\mathrm{d}}$ & $1.81 \pm 4.28(0-3)$ & $1.03 \pm 2.92(0-2)$ & 0.000 \\
CDAI $^{\mathrm{e}}$ & $6.4 \pm 2.7(3-7)$ & $4.07 \pm 2.18(3-5)$ & 0.000 \\
\hline
\end{tabular}

CDAI: Clinical Disease Activity Indices ${ }^{a} P<0.05$ is significant.

$\mathrm{b}$ (none: 0 , low grade: 1 , high grade: 2 .)

${ }^{\mathrm{c}}$ (none $=0$, mild: 1 , moderate: 2 , severe: 3 .)

d (0: none, $1: \leq 30 \mathrm{~min}, 2: 30-60 \mathrm{~min}, 3: 1-2 \mathrm{~h}, 4: 2-4 \mathrm{~h}, 5: \geq 4 \mathrm{~h}$ and $6:$ all day.)

${ }^{\mathrm{e}}($ Remission $\leq 2.8$, low disease activity $\leq 10$, moderate disease activity $\leq 22$, high disease activity $>22$.

TABle 4: Comparison between variable at the third and sixth month. (end month of each trimester).

\begin{tabular}{lccc}
\hline Variable & Morning group \pm SD $(\min -\max )$ & Bedtime group \pm SD $(\min -\max )$ & $P$ value \\
\hline Fatigue score & $4.37 \pm 1.44(2-7)$ & $2.57 \pm 1.00(1-6)$ & $<0.0001$ \\
Morning stiffness score & $0.77 \pm 0.33(1-3)$ & $0.74 \pm 0.51(1-3)$ & 0.0003 \\
CDAI & $6.20 \pm 1.87(4-8)$ & $4.02 \pm 2.00(3-7)$ & $0.82 \pm 0.54(0-2)$ \\
Pain score & $0.85 \pm 0.55(0-2)$ & $6.4 \pm 0.76(6-23)$ & 0.7367 \\
CRP $(\mathrm{mg} / \mathrm{dl})$ & $7.6 \pm 0.93(6-26)$ & $17 \pm 8.93(2-40)$ & 0.04 \\
ESR $(\mathrm{mm} / \mathrm{h})$ & $18 \pm 11.56(1-56)$ & & 0.283 \\
\hline
\end{tabular}

ESR: erythrocyte sedimentation rate; CRP: C reactive protein.

TABLE 5: Comparison between adverse effects in two groups.

\begin{tabular}{lcc}
\hline Adverse effects & $\begin{array}{c}\text { Morning group } \\
\text { number (percent) }\end{array}$ & $\begin{array}{c}\text { Night group } \\
\text { number } \\
\text { (percent) }\end{array}$ \\
\hline Nocturia & No & $3(5 \%)$ \\
Sleep disturbance & No & $1(0.016 \%)$ \\
BP disturbance & $2(0.03 \%)$ & No \\
Tachycardia & $2(0.03 \%)$ & $2(0.03 \%)$ \\
Overt infection & No & No \\
\hline
\end{tabular}

study. Baseline characteristics of the patients are shown in Table 1. Disease duration among our patients was between 12 months to 40 years with mean of $7.1 \pm 7.5$ years as mentioned in Table 1. But they were on DMARDs from beginning of disease in some and months after initiation of disease in the others. Minimum duration of DMARD used in our patient when they enrolled in the study was around 6 months. Thirty-seven patients (53.3\%) were treated with two DMARDs, and ten (16.7\%) patients were treated with three DMARDs (Table 2).

The mean of the morning stiffness was decreased and had significant statistical differences $(P$ value $=0.000)$. The mean of the CDAI was reduced at the end of the second three months, when the patients were treated with prednisolone at night $(P$ value $=0.000)$. The mean of the fatigue score and CRP level were decreased when prednisolone was administrated at $10 \mathrm{PM}$, and differences were significant $(P$ value $<0.05$ ). The mean of the pain score was decreased when the patients took prednisolone at night, but there was no significant statistical difference $(P$ value $=0.146)$. Also, the mean of tender joint was decreased when the patient took prednisolone at nigh; however, there was no significant statistical difference $(P$ value $=0.129)$. The mean of the ESR was decreased, but the difference was not significant $(P$ value $=0.283$ ). These data are compared in Table 3 .

Means of each variable at the end of the first and the second trimester are compared in Table 4.

Nocturia was seen in 3 patients (5\%) when prednisolone was administrated at night, and sleep disturbance was seen in 1 patient in this group (Table 5).

In Figure 1, comparison of variable means at baseline and end of before and after phase was shown.

\section{Discussion}

Better control of inflammation and improving patient symptoms related to inflammatory state with minimal drug adverse effect is the best management in chronic inflammatory conditions like RA.

In the current study, administration of prednisolone at night induced a significant beneficial effect on the morning stiffness $(P<0.001)$, which was similar to the results of the Arvison [8] and Buttgereit report [13]. It should be stated that in the Arvidson study, prednisolone was taken at $2 \mathrm{AM}$, which could potentially have a negative effect on the sleep pattern of patients [8]. De Silva et al. showed a significantly shorter duration of morning stiffness in patients when prednisolone was given at night [14]. Similar to our study, the patients were on oral prednisolone at 10 PM Deandrade et al. also noted that a small evening dose was more effective than the same dose given in the morning [15]. Klinefelter and his coworkers evaluated the safety of single low-dose prednisone therapy. In their study, a number of patients required nocturnal doses to control their morning stiffness and did not show any more suppression of the HPA axis suppression than the patients receiving morning doses [16]. 


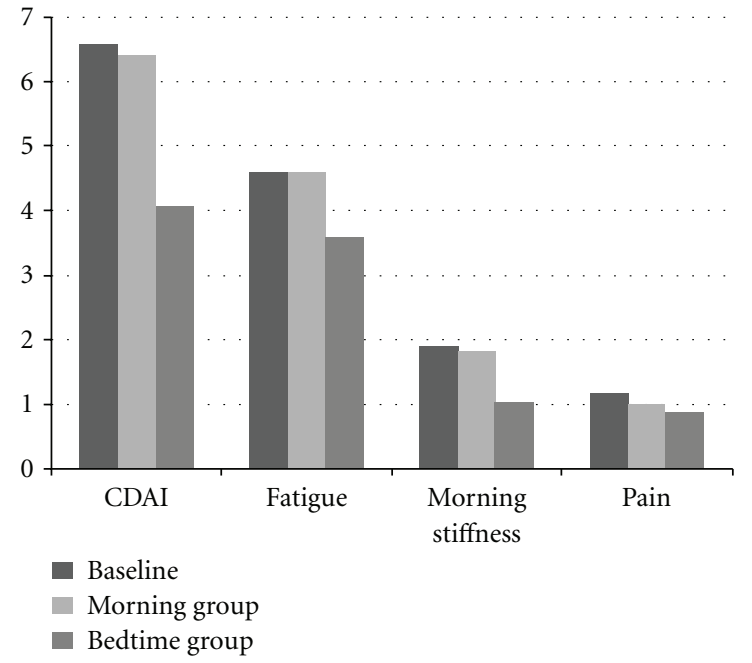

FIGURE 1: Diagram showing mean of variables in baseline, morning, and bedtime group.

Fatigue is frequently identified as a significant symptom by RA patients [17]. The prevalence of clinically relevant fatigue is commonly seen between $40 \%$ and $80 \%$ of patients with RA [18]. We showed that the mean of fatigue scores also decreased when the prednisolone was administrated at night $(P<0.000)$. However, this variable was not taken into consideration in the previous studies, although it is a main complaint of patients.

The theoretical optimal timing of prednisolone administration could be at $2 \mathrm{AM}$ but due to interfering with predisturbed sleep in RA patients, modified release prednisolone was introduced to overcome this problem. We, in our study, showed that shifting single oral prednisolone from morning to bedtime schedule also could have similar beneficial effect on morning symptom which is universally available.

In our patients, there was a tendency for a decrease in the mean of pain scores when the patients took prednisolone at night, although employing Wilcoxon's test showed no statistical significant difference. Result of means of variables was similar within and at the end of each trimester.

However, in the study conducted by Arvidson [8] and Buttgereit [13], pain scores decreased statistically significant after bedtime administration of GCs.

In another study, clinical outcome after administration of prednisolone at $8 \mathrm{AM}, 1 \mathrm{PM}$ and $11 \mathrm{PM}$ was compared. Four weeks later, there was no difference in pain or stiffness scores between these times of prednisolone administration [19]. Also, Karatay and his colleagues reported that there were no differences between pain and morning stiffness scores when GCs were administered at different times of the day [20]. The differences between our and their findings could be attributed to the total daily doses and/or ethnic differences in the patients.

A comparison of objective measurements of joint inflammation revealed no significant statistical differences, although there was a decline in the number of tender joints.
Nevertheless, it should be mentioned that in most of our patients, joint tenderness was not a predominant symptom.

In our study, CDAI was also used for clinical assessment and there was a significant statistical difference between the mean of two groups.

In spite of a reduction in the mean of ESR, there was no significant statistical difference $(P=0.283)$. As in the studies done by Arvidson et al. and Karatay et al., the mean of CRP levels decreased significantly following nocturnal use of prednisolone $[8,20]$.

We emphasized that adverse profile of shifting prednisolone from morning to bedtime was negligible in our survey and none of them was related to HPA axis derangement.

Addressing to our limitations in the study, we should mention that, due to clinical nature of our study we did not determine the cytokines such as $\mathrm{IL}_{6}$ and hormones involving in HPA axis notably serum and urine cortisol that it could be designed as a separate supplementary study. Also, we think that due to potential beneficial of bedtime dosing of prednisolone, we could test smaller doses of prednisolone at bedtime along with this study and compare the mean of prednisolone doses in two phases (groups) of study.

\section{Conclusion}

In conclusion, our study showed that similar doses of prednisolone could be safely administrated at bedtime with better clinical responses in most (but not all) of RA patients.

\section{Conflict of Interests}

The authors declare that they have no conflict of interests.

\section{Acknowledgments}

The authors thank Dr. F. Alam-Rajabi and Dr. Z. Mehrnahad for their contribution in data gathering and Dr. H. Lotfi for statistical analysis.

\section{References}

[1] M. Cutolo, A. Sulli, C. Pizzorni et al., "Circadian rhythms: glucocorticoids and arthritis," Annals of the New York Academy of Sciences, vol. 1069, pp. 289-299, 2006.

[2] M. Cutolo, B. Villaggio, K. Otsa, O. Aakre, A. Sulli, and B. Seriolo, "Altered circadian rhythms in rheumatoid arthritis patients play a role in the disease's symptoms," Autoimmunity Reviews, vol. 4, no. 8, pp. 497-502, 2005.

[3] M. Cutolo, K. Otsa, O. Aakre, and A. Sulli, "Nocturnal hormones and clinical rhythms in rheumatoid arthritis," Annals of the New York Academy of Sciences, vol. 1051, pp. 372381, 2005.

[4] R. H. Straub and M. Cutolo, "Circadian rhythms in rheumatoid arthritis: implications for pathophysiology and therapeutic management," Arthritis and Rheumatism, vol. 56, no. 2, pp. 399-408, 2007.

[5] M. Cutolo, B. Seriolo, C. Craviotto, C. Pizzorni, and A. Sulli, "Circadian rhythms in RA," Annals of the Rheumatic Diseases, vol. 62, no. 7, pp. 593-596, 2003. 
[6] J. R. Kirwan, L. Clarke, L. P. Hunt, M. G. Perry, R. H. Straub, and D. S. Jessop, "Effect of novel therapeutic glucocorticoids on circadian rhythms of hormones and cytokines in rheumatoid arthritis: annals of the New York Academy of Sciences," Annals of the New York Academy of Sciences, vol. 1193, pp. 127$133,2010$.

[7] N. G. Arvidson, B. Gudbjornsson, L. Elfman, A. C. Ryden, T. H. Totterman, and R. Hallgren, "Circadian rhythm of serum interleukin-6 in rheumatoid arthritis," Annals of the Rheumatic Diseases, vol. 53, no. 8, pp. 521-524, 1994.

[8] N. G. Arvidson, B. Gudbjörnsson, A. Larsson, and R. Hällgren, "The timing of glucocorticoid administration in rheumatoid arthritis," Annals of the Rheumatic Diseases, vol. 56, no. 1, pp. 27-31, 1997.

[9] I. C. Kowanko, R. Pownall, and M. S. Knapp, "Time of day of prednisolone administration in rheumatoid arthritis," Annals of the Rheumatic Diseases, vol. 41, no. 5, pp. 447-452, 1982.

[10] M. Cutolo, R. H. Straub, and F. Buttgereit, "Circadian rhythms of nocturnal hormones in rheumatoid arthritis: translation from bench to bedside," Annals of the Rheumatic Diseases, vol. 67, no. 7, pp. 905-908, 2008.

[11] D. Aletaha, T. Neogi, A. J. Silman et al., "2010 Rheumatoid arthritis classification criteria: an American College of Rheumatology/European League against rheumatism collaborative initiative," Arthritis and Rheumatism, vol. 62, no. 9, pp. 2569-2581, 2010.

[12] J. S. Smolen, F. C. Breedveld, M. H. Schiff et al., "A simplified disease activity index for rheumatoid arthritis for use in clinical practice," Rheumatology, vol. 42, no. 2, pp. 244-257, 2003.

[13] F. Buttgereit, G. Doering, A. Schaeffler et al., "Efficacy of modified-release versus standard prednisone to reduce duration of morning stiffness of the joints in rheumatoid arthritis (CAPRA-1): a double-blind, randomised controlled trial," The Lancet, vol. 371, no. 9608, pp. 205-214, 2008.

[14] M. De Silva, A. Binder, and B. L. Hazleman, "The timing of prednisolone dosage and its effect on morning stiffness in rheumatoid arthritis," Annals of the Rheumatic Diseases, vol. 43, no. 6, pp. 790-793, 1984.

[15] J. R. Deandrade, J. N. McCormick, and A. G. Hill, "Small doses of prednisolone in the management of rheumatoid arthritis," Annals of the Rheumatic Diseases, vol. 23, pp. 158-162, 1964.

[16] H. F. Klinefelter, W. L. Winkenwerder, and T. Bledsoe, "Single daily dose prednisone therapy," Journal of the American Medical Association, vol. 241, no. 25, pp. 2721-2723, 1979.

[17] S. Stebbings, P. Herbison, T. C. H. Doyle, G. J. Treharne, and J. Highton, "A comparison of fatigue correlates in rheumatoid arthritis and osteoarthritis: disparity in associations with disability, anxiety and sleep disturbance," Rheumatology, vol. 49, no. 2, pp. 361-367, 2009.

[18] F. Wolfe, D. J. Hawley, and K. Wilson, "The prevalence and meaning of fatigue in rheumatic disease," Journal of Rheumatology, vol. 23, no. 8, pp. 1407-1417, 1996.

[19] I. C. Kowanko, R. Pownall, and M. S. Knapp, "Time of day of prednisolone administration in rheumatoid arthritis," Annals of the Rheumatic Diseases, vol. 41, no. 5, pp. 447-452, 1982.

[20] S. Karatay, K. Şenel, M. Uğur, and K. Yildirim, "The timing of low dose glucocorticoid therapy in treatment of rheumatoid arthritis," Pain Clinic, vol. 13, no. 4, pp. 305-312, 2002. 

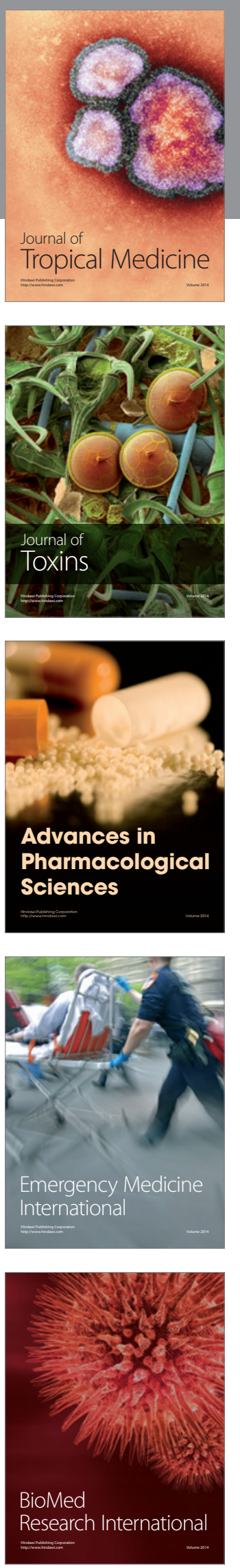
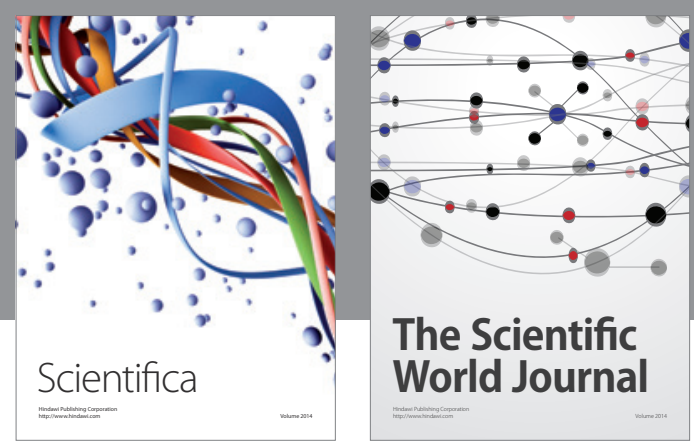

The Scientific World Journal
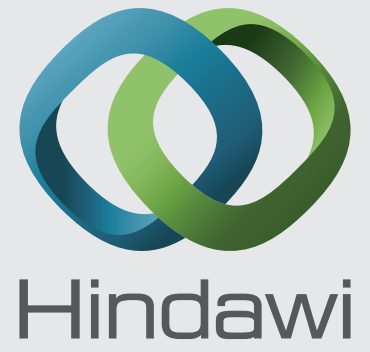

Submit your manuscripts at

http://www.hindawi.com
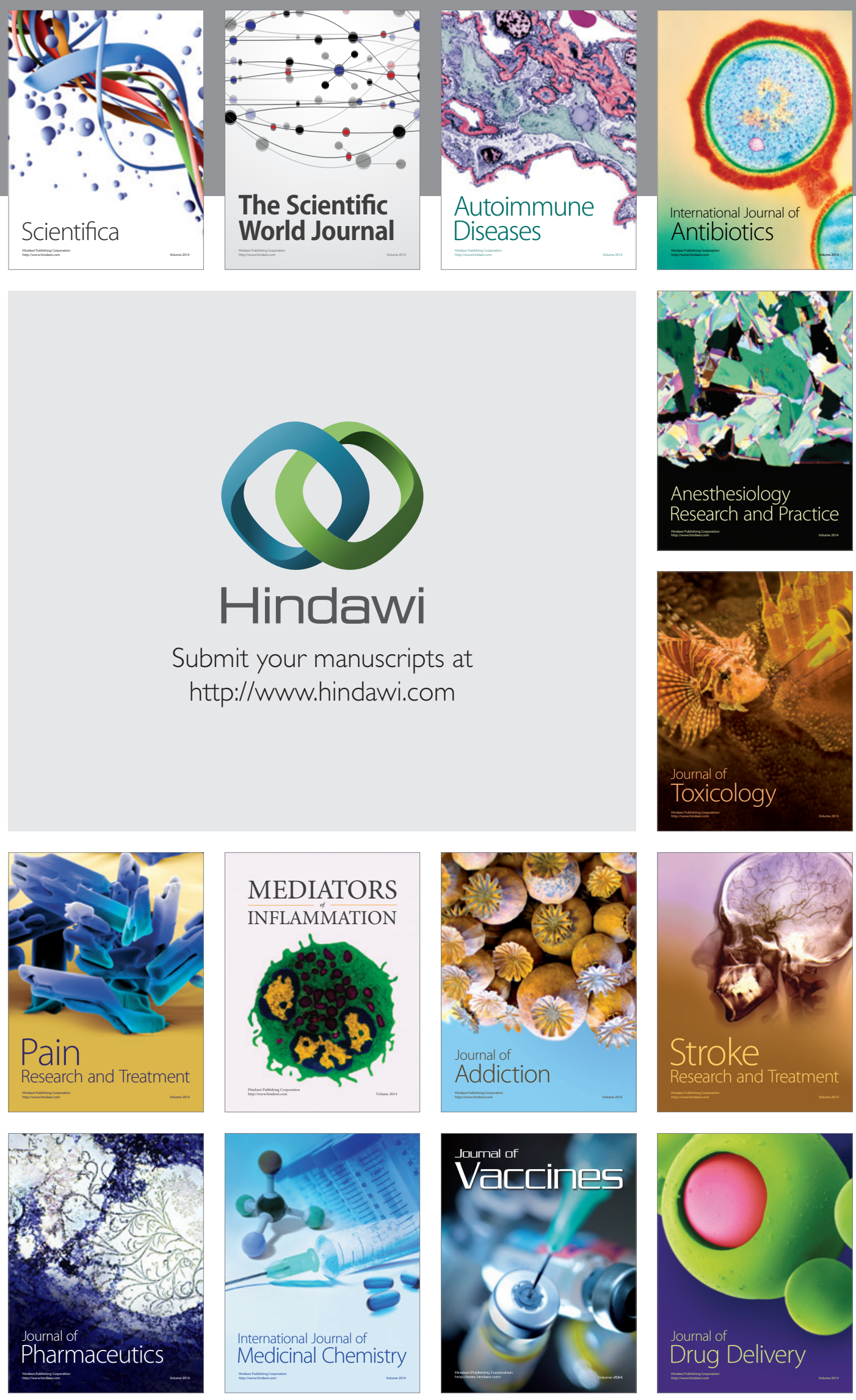